\title{
Evaluación por inmunohistoquímica de la expresión de hormonas hipofisiarias y del marcador de proliferación celular Ki-67 en tejido de adenomas causantes de acromegalia
}

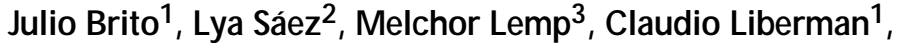 \\ Harold Michelsen ${ }^{1}$, A Verónica Araya ${ }^{1}$. \\ Immunohistochemistry for pituitary \\ hormones and Ki-67 in growth \\ hormone producing pituitary adenomas
}

Background: Growth hormone (GH) producing adenomas, frequently express several hormones. This condition could confer them a higher proliferative capacity. Ki-67 is a nuclear protein antigen that is a marker for proliferative activity. Aim: To measure the immunohistochemical hormone expression in pituitary adenomas, excised from patients with acromegaly. To determine if the plurihormonal condition of these adenomas is associated with a higher proliferative capacity, assessed through the expression of Ki-67. Material and methods: Forty one paraffin embedded surgical samples of pituitary adenomas from patients with acromegalia were studied. Immunohistochemistry for $\mathrm{GH}$, prolactin (PRL), follicle stimulating hormone (FSH), luteinizing hormone ( $\mathrm{LH}$ ), thyroid stimulating hormone (TSH), adrenocorticotropin (ACTH) and for the expression of Ki-67 was carried out. Results: All samples were positive for GH. Twenty seven had positive staining for PRL, 12 had positive staining for glycoproteic hormones and 11 for PRL and one or more glycoproteic hormones. Mean staining for Ki-67 was $2.6 \pm 3.3 \%$. There were no differences in the expression of this marker between mono or plurihormonal tumors. The expression was neither associated with extrasellar extension. Conclusions: Half of $\mathrm{GH}$ producing pituitary adenomas are plurihormonal. There are no differences in the expression of Ki-67 between mono and plurihormonal adenomas (Rev Méd Chile 2008; 136: 831-6).

(Key words: Acromegaly; Ki-67 protein, human; Human growth hormone)

Recibido el 10 de octubre, 2007. Aceptado el 27 de marzo, 2008.

${ }^{1}$ Sección Endocrinología, Departamento de Medicina; ${ }^{2}$ Departamento de Anatomía Patológica; ${ }^{3}$ Departamento de Neurología y Neurocirugía del Hospital Clínico de la Universidad de Chile.

Correspondencia a: Dra. Verónica Araya Q. Sección de Endocrinología, Hospital Clínico de la Universidad de Chile. Santos Dumont 999, Independencia. Santiago, Chile. Fax: 56-2-7776891. E mail: varaya@redclinicauchile.cl 
L a acromegalia es el cuadro clínico derivado del exceso de hormona de crecimiento o somatotropina $(\mathrm{GH})$, secretada por un tumor de localización hipofisiaria en más de $95 \%$ de los casos ${ }^{1}$.

Sólo $60 \%$ de los tumores provenientes de pacientes con acromegalia corresponden a adenomas somatotropos puros, $25 \%$ son adenomas mixtos de células somato y mamotropas, $10 \%$ son adenomas de células mamosomatotropas y 5\% corresponde a adenomas acidófilos derivados de un stem cell primordial y que representaría al progenitor común de las células de $\mathrm{GH}$ y prolactina $(\mathrm{PRL})^{2-5}$.

La caracterización inmunohistoquímica y estudios ultraestructurales han permitido demostrar que los adenomas hipofisiarios de pacientes acromegálicos no sólo expresan y secretan $\mathrm{GH}$, sino que también muestran localización concomitante de otras hormonas como PRL u hormonas glicoproteicas como la tiroestimulante (TSH), foliculoestimulante (FSH) o luteinizante $(\mathrm{LH})^{6,7}$. Con respecto a la citogénesis de los adenomas plurihormonales, se ha sugerido que ellos pueden derivar de un stem cell primordial plurihormonal que está sometido a una diferenciación multidireccional y es capaz de producir más de una hormona ${ }^{8,9}$. Probablemente, el factor transcripcional específico de la hipófisis Pit-1, que regula la síntesis de GH, PRL y TSH, esté involucrado en la citodiferenciación de estos adenomas ${ }^{10}$.

La expresión de GH/PRL conferiría una mayor capacidad tumorigénica de las células de la hipófisis anterior. En cambio, la presencia simultánea en tumores de GH y subunidad alfa de hormonas glicoproteicas puede corresponder a la proliferación de una subpoblación normal de células de la hipófisis anterior ${ }^{11}$.

Algunos aspectos histológicos, como la distribución intracelular de citokeratina, la densidad de los gránulos y la presencia de Ki-67, se han asociado a recurrencia y capacidad de invasión de los adenomas hipofisiarios, principalmente hacia el seno cavernoso ${ }^{2,12}$.

El Ki-67 es un antígeno proteico nuclear expresado en todo el ciclo celular (G1, M, G2, y $\mathrm{S})$, excepto en G0. A diferencia del índice mitótico que sólo evidencia división celular actual, el Ki-67 es un fiel marcador de la actividad proliferativa. Su expresión sería directamente proporcional al tamaño, capacidad de invasión y recidiva de adeno- mas hipofisiarios ${ }^{13,14}$. Un aspecto interesante es su correlación con el oncogén subtipo 4 de receptor de factor de crecimiento fibroblástico, FGFR4, cuya expresión en tejido hipofisiario ha sido relacionada con proliferación celular y crecimiento tumoral ${ }^{15}$.

Como existe un alto porcentaje de pacientes acromegálicos que no logran curación bioquímica luego de una primera intervención neuroquirúrgi$\mathrm{ca}^{16}$, y que requerirán una segunda cirugía u otras terapias para conseguir este objetivo, a los clásicos factores de riesgo de fracaso quirúrgico, como la edad al momento del diagnóstico, tamaño, extensión tumoral y concentración de GH inicial, sería interesante incorporar algunos aspectos inmunohistoquímicos de los adenomas.

En nuestro medio no se dispone de información acerca de la expresión de las diferentes hormonas hipofisiarias y de Ki-67 en adenomas hipofisiarios, por lo cual, los objetivos de nuestro estudio fueron: evaluar en adenomas secretores de GH provenientes de pacientes intervenidos por acromegalia, la expresión de las hormonas de la hipófisis anterior por técnica de inmunohistoquímica; establecer si la condición plurihormonal se asocia con un mayor índice de proliferación del marcador Ki-67 y evaluar si existen diferencias clínicas según la expresión de otras hormonas y de Ki-67.

\section{MATERIAL Y MÉTODO}

Del archivo de Anatomía Patológica del Hospital Clínico de la Universidad de Chile se rescataron las muestras quirúrgicas incluidas en parafina de 45 pacientes operados en el Servicio de Neurocirugía entre los años 1985 y 2004, por el diagnóstico de acromegalia documentado por prueba de tolerancia a la glucosa. Después de la revisión de las placas histológicas se descartaron 4 (2 hipófisis normales, 1 con fibrosis isquémica y 1 muestra no diagnóstica). Además, se revisó la ficha clínica de cada caso. En todos ellos la cirugía fue el primer tratamiento.

En las 41 muestras en las que se identificó tejido tumoral, se investigó por técnica de inmunohistoquímica la localización concomitante de GH, PRL, FSH, LH, TSH, ACTH y también, la expresión de Ki-67. La indemnidad del tejido y la calidad de la tinción inmunohistoquímica, fueron certificadas por un patólogo experto. 
Las muestras del tejido incluido fueron cortadas en secciones de $4 \mu \mathrm{m}$, desparafinadas y rehidratadas con agua destilada. Se incubaron en presencia de anticuerpo primario por $30 \mathrm{~min}$ a temperatura ambiente. El set de anticuerpos usados fue anti GH, PRL, FSH, LH, TSH (Biosgenes), anti ACTH (Neomarkers), Ki-67 (Dakocitomation). Enseguida se incubaron por 20 min en presencia de anticuerpo secundario biotinilado y, posteriormente, se agregó estreptavidina-peroxidasa de rábano picante, para finalmente revelar con solución cromógena DAB (3,3'-diaminobenzidina), por un máximo de 20 min. La actividad peroxidasa intrínseca fue previamente bloqueada por incubación en peróxido de hidrógeno al 3\% por 3 min. Se realizó tinción suave de núcleos con hematoxilina-eosina.

Para medir la presencia de Ki-67, se realizó primariamente la recuperación de los antígenos nucleares, mediante incubación en buffer TrisEDTA, a pH 9, en microondas por 6 min a potencia máxima y $1 \mathrm{~h}$ a baja potencia. Se consideró positiva la presencia de marcación para Ki-67 en citoplasma difuso o Golgi, examinando el área más densa, en donde se contaron 100 a 200 núcleos por campo, dando un porcentaje en base al recuento. Si en 500 núcleos aparece escasa marca, se considera $<1 \%$. Según se define en la literatura, una marcación inferior a 5\% es considerada baja y una marcación $>10 \%$ de las células es un alto índice de proliferación y se relaciona con capacidad de invasión ${ }^{17}$.

El examen microscópico se realizó con aumento de $400 \mathrm{x}$, usando grilla ocular, en un microscopio NIKON Alphaphot 2.

Este protocolo de estudio fue aprobado por el Comité de Ética del Hospital Clínico de la Universidad de Chile.

Estadística. Los resultados se presentan como promedio \pm desviación estándar. Se utilizaron los tests de Mann-Whitnay y $\chi^{2}$ con corrección de Yates para evaluar las diferencias entre medias y proporciones respectivamente. Se consideró una diferencia significativa a un $\mathrm{p}<0,05$.

\section{Resultados}

De los 41 tumores, sólo 4 correspondieron a microadenomas. En todos ellos se observó tinción positiva (+) para GH. Catorce (34\%) fueron monohormonales para GH y en 27 (66\%) se observó además tinción $(+)$ para una o más hormonas. En la Tabla 1 se observan las combinaciones presentes en todos los adenomas con sus respectivos porcentajes.

En la Tabla 2 se presentan las características clínicas de los pacientes con adenomas monohormonales y los con adenomas plurihormonales. No hubo diferencia significativa para sexo, edad 0 tiempo de evolución de la enfermedad. Tampoco hubo diferencia significativa entre los grupos para los niveles de GH y PRL preoperatorios. Los niveles séricos de TSH, FSH y LH estaban dentro del rango normal en todos los casos. El porcentaje de casos que presentó expansión extraselar (supra o paraselar) en la tomografía axial computada (TAC) o resonancia nuclear magnética (RNM) de silla turca, fue similar en los dos grupos. No hubo diferencia en el porcentaje de curación, entre el grupo monohormonal y el grupo plurihormonal.

Inmunohistoquímica para Ki-67. En 32 de los 41 tumores, se obtuvo muestra suficiente para realizar la tinción inmunohistoquímica para Ki-67.

El valor promedio del porcentaje de marcación Ki-67 fue $2,64 \pm 3,3 \%$, rango $1 \%$ a $15 \%$.

No se encontró una diferencia significativa entre los adenomas monohormonales y los plurihormonales para el porcentaje de marcación promedio de Ki67 (Tabla 3) pero, los 3 tumores que presentaron un porcentaje mayor o igual a $10 \%$ fueron plurihormonales (un tumor GH/FSH(+)/PRL (-) y 2 tumores GH/PRL (+)). Los 3 comespondieron a macroadeno-

\section{Tabla 1. Patrones de inmunohistoquímica en 41 adenomas hipofisiarios secretores de $\mathbf{G} \mathbf{H}$}

\begin{tabular}{|lrr|}
\hline Hormonas & $\mathrm{n}$ & $\%$ \\
\hline GH & 14 & 34,1 \\
GH + PRL & 4 & 9,8 \\
GH + FSH & 9 & 22,0 \\
GH + TSH & 1 & 2,4 \\
GH + PRL + FSH & 8 & 19,5 \\
GH + FSH + TSH & 1 & 2,4 \\
GH + PRL + FSH + TSH & 3 & 7,3 \\
GH + FSH + TSH + LH & 1 & 2,4 \\
Total & 41 & 100 \\
\hline
\end{tabular}


Tabla 2. Características clínicas en $\mathbf{4 1}$ pacientes portadores de adenomas hipofisiarios secretores de $\mathbf{G} \mathbf{H}$, comparados según la presencia de inmunotinción para otras hormonas en el tejido resecado

\begin{tabular}{|lll|}
\hline & $\begin{array}{l}\text { GH monohormonal } \\
\mathrm{n}=14\end{array}$ & $\begin{array}{l}\text { Plurihormonal } \\
\mathrm{n}=27\end{array}$ \\
\hline Sexo (H/M) & $4 / 10$ & $9 / 18$ \\
Edad (años) & $45,9 \pm 11,5(33-65)$ & $44,3 \pm 14(16-65)$ \\
Tiempo de evolución (años) & $6,8 \pm 8,2(0,5-15)$ & $6,6 \pm 8,2(0,5-30)$ \\
GH basal (ng/ml) & $30,7 \pm 23,4(7-81)$ & $28,9 \pm 24,3(4,8-125)$ \\
PRL basal (ng/ml) & $33,9 \pm 37,8(2,1-122,8)$ & $46,2 \pm 73,7(3,1-330,5)$ \\
Expansión extraselar & $10 / 14(71,4 \%)$ & $12 / 27(44,4 \%)$ \\
$\%$ Curación & $4 / 14(28,6 \%)$ & $5 / 27(18,5 \%)$ \\
\hline
\end{tabular}

Los resultados se presentan como promedio \pm DS. No se observó diferencia estadísticamente significativa entre los parámetros evaluados.

mas y el tiempo promedio de evolución del cuadro clínico de los pacientes fue 1,5 años. El porcentaje de marcación Ki-67 más alto (15\%) se observó en el tumor del paciente más joven de la serie (16 años), que curó después de una segunda intervención quirúrgica y radioterapia.

Al comparar los tumores con y sin inmunotinción presente para PRL, y los con y sin expansión extraselar, tampoco observamos una diferencia significativa en el porcentaje de marcación de Ki67 (Tabla 3).

\section{DisCUSIÓN}

En este estudio demostramos que más de $50 \%$ de los adenomas causantes de acromegalia de nues- tra serie presentó inmunotinción positiva para otra(s) hormona(s) de la hipófisis anterior, además de $\mathrm{GH}$, lo que concuerda con lo descrito previamente en la literatura sobre la alta frecuencia de la característica plurihormonal de estos adenomas hipofisiarios $5,6,18$.

En nuestra serie no observamos diferencia en los niveles de GH sérica ni en el porcentaje de adenomas con expansión extraselar entre los tumores plurihormonales y los monohormonales. Otros autores han demostrado que los niveles de GH en plasma se correlacionan en forma inversa con el porcentaje de inmunomeactividad para $\mathrm{GH}$ presente en el tejido, pero no han encontrado asociación entre la característica plurihormonal y el tamaño del tumor ${ }^{18}$. Otros han postulado que los adenomas GH/PRL (+) tendrían una mayor capacidad prolifera-

\section{Tabla 3. Porcentaje de marcación Ki-67 en tejido de 32 adenomas hipofisiarios con inmunotinción positiva para G H}

\begin{tabular}{|lcc|}
\hline & $\mathrm{n}$ & \% de marcación Ki-67 \\
\hline GH monohormonal & 10 & $1,8 \pm 1,7$ \\
Plurihormonal & 22 & $3 \pm 3,8$ \\
Según inmunotinción para PRL & 12 & $3,2 \pm 4,1$ \\
$\quad$ PRL (+) & 20 & $2,2 \pm 2,4$ \\
$\quad$ PRL (-) & 17 & $2,58 \pm 3,9$ \\
Según expansión extraselar (ES) & 15 & $2,73 \pm 2,57$ \\
$\quad$ Con ES & 15 & \\
Sin ES & \\
\hline
\end{tabular}

No se observó diferencia estadísticamente significativa entre los grupos analizados. 
tiva y que la expresión de hormonas glicoproteicas pudiera representar un estadio más diferenciado $\mathrm{y}$, por ende, menos proclive a la proliferación ${ }^{10}$. En los casos evaluados, no observamos diferencias en la proporción de adenomas con tinción para PRL y sin tinción para PRL, que presentaron expansión extraselar en la TAC o RNM de silla turca, ni en el porcentaje de marcación de Ki-67 en ambos grupos de adenomas. Tampoco hubo diferencia en el promedio de la PRL sérica (datos no mostrados). Este hallazgo es similar a lo descrito por otros autores, que han demostrado que los adenomas mamosomatotropos o mixtos somatotropos-lactotropos, se manifiestan clínicamente por acromegalia con niveles de PRL normales en la mayonía de los casos $^{5,18,19}$. Aparentemente, la inmunorreactividad para una hormona específica no necesariamente se asocia con el aumento de los niveles séricos o con la clínica correspondiente probablemente, porque secretan hormonas biológicamente inactivas.

El antígeno nuclear Ki-67 ha sido reconocido como un marcador de proliferación celular y su expresión sería directamente proporcional a la capacidad de invasión y recidiva tumoral ${ }^{20,21}$. En adenomas hipofisiarios se ha descrito un porcentaje de tinción Ki-67 entre 1,1\% y 5,5\%. Para los tumores secretores de $\mathrm{GH}$, se describió en dos series $0,92 \%$ y 2,18\% 20,21 , respectivamente. En nuestra serie, el porcentaje de marcación promedio fue levemente superior, 2,64\% y en los adenomas plurihormonales observamos una tendencia a ser mayor a 3\%. Incluso 3 de ellos presentaron un porcentaje de tinción Ki-67 igual o superior a 10\%, lo que corresponde a un alto índice de proliferación de acuerdo a lo descrito por Thapar et $\mathrm{al}^{22}$. Un aspecto interesante fue que estos casos correspondieron a macroadenomas, en los cuales el tiempo de evolución transcurrido desde el inicio de la sintomatología hasta el momento del diagnóstico no superó los dos años. Aunque el número de muestras analizadas es reducido para aseverar que

\section{REFERENCIAS}

1. AACE Acromegaly Guidelines Task Force. American Association of Clinical Endocrinologists medical guidelines for clinical practice for the diagnosis and treatment of acromegaly. Endocrine Practice 2004; 10: 213-25. la condición plurihormonal, asociada a un elevado porcentaje de Ki-67, confiere mayor capacidad de proliferación y un mayor riesgo de crecimiento e invasión tumoral, esta observación es altamente sugerente y se debería confirmar con el análisis futuro de un mayor número de adenomas.

En la literatura aún existen controversias respecto a si Ki-67 es un marcador biológico confiable para predecir la capacidad de proliferación y recidiva en adenomas hipofisiarios, ya que se ha observado una superposición entre adenomas indolentes y tumores invasores ${ }^{2,20-24}$. Sin embargo, hay que señalar que estas series están compuestas mayoritariamente por adenomas no funcionantes y que no se ha analizado dirigidamente a los adenomas secretores de GH. Además, es importante tener en cuenta al comparar resultados cuantitativos, las diferencias en la sensibilidad de los diferentes kits de anticuerpos para el antígeno Ki-6725.

Concluimos que los adenomas secretores de GH son plurihormonales en más de $50 \%$ de los casos. Aunque no fue demostrado estadísticamente, los adenomas plurihormonales presentan una tendencia a un mayor índice de proliferación evaluado con el antígeno nuclear Ki-67. Se requiere analizar un mayor número de muestras para establecer su utilidad en la caracterización inmunohistoquímica de los adenomas secretores de GH y en el pronóstico de los pacientes con acromegalia.

\section{Agradecimientos}

Agradecemos a la Oficina de Apoyo a la Investigación Clínica (OAIC) del Hospital Clínico de la Universidad de Chile y al Laboratorio Chile por ayudarnos con el financiamiento de los reactivos necesarios para el estudio. También agradecemos al Sr. Gabriel Sánchez Jorquera por su colaboración desinteresada en la realización de las inmunohistoquímicas y a todos los miembros de la sección de Endocrinología que han sido los médicos tratantes de estos pacientes.

2. At-Shraim M, Asa SL. The 2004 World Health Organization classification of pituitary tumors: What is new? Acta Neuropathol 2006; 111: 1-7.

3. Matsuno A, Teramoto A, TAKekoshi S, Sanno N, Osamura RY, Kirino T. Expression of plurihormonal mRNAs in somatotrophic adenomas detected using a nonisotopic in situ hybridization method: compari- 
son with lactotrophic adenomas. Hum Pathol 1995; 26: 272-9.

4. Sakharova AA, Dimaraki EV, Chandler WF, Barkan AL Clinically silent somatotropinomas may be biochemically active. J Clin Endocrinol Metab 2005; 90: 2117-21.

5. Ho DM, Hsu CY, Ting LT, Chiang H. Plurihormonal pituitary adenomas: immunostaining of all pituitary hormones is mandatory for correct classification. Histopathology 2001; 39: 310-9.

6. Asa SL, Kovacs K, Horvath E, Losinski NE, Laszlo FA, Dомокоs I ET AL. Human fetal adenohypophysis. Electrón microscopic and ultrastructural immunocytochemical analysis. Neuroendocrinology 1988; 48: 423-31.

7. Matsuno A, Teramoto A, Takekoshi S, Sanno $\mathrm{N}$, Osamura RY, KirINo T. HGH, PRL, and ACTH gene expression in clinically nonfunctioning adenomas detected with nonisotopic in situ hybridization method. Endocr Pathol 1995; 6: 13-20.

8. Kamtani $\mathrm{H}$, Masuzawa $\mathrm{H}$, Kanazawa I, Kubo T. The multihormonal character of pituitary adenomas: Immuno-electron microscopic studies. Neuropathology 1999; 19: 40-50.

9. Mccomb DJ, Bayley TA, Horvath E, Kovacs K, Kourides IA. Monomorphous plurihormonal adenoma of the human pituitary. A histologic, immunocytologic and ultrastructural study. Cancer 1984; 53: 1538-44.

10. Herman V, Fagin J, Gonsky R, Kovacs K, Melmed S. Clonal origin of pituitary adenomas. J Clin Endocrinol Metab 1990; 71: 1427-33.

11. Inada K, Oda K, Utsunomiya $H$, Itoh J, Osamura RY. Immunohistochemical analysis of GH-producing adenomas with special emphasis on plurihormonality of individual tumor cells by double staining. Tokai J Exp Clin Med 1992; 17: 213-22.

12. Prevedello DM, Jagannathan J, Jane JA Jr, Lopes MB, Laws ER JR. Relevance of high Ki-67 in pituitary adenomas. Case report and review of the literature. Neurosurg Focus 2005; 19: E11.

13. Zhao D, Tomono Y, Nose T. Expression of P27kip1 and Ki-67 in pituitary adenomas: an investigation of marker of adenoma invasiveness. Acta Neurochir (Wien) 1999; 141: 187-92.

14. Pan LX, Chen ZP, Liu YS, Zhao JH. Magnetic resonance imaging and biological markers in pituitary adenomas with invasion of the cavernous sinus space. J Neurooncol 2005; 74: 71-6.

15. Qian ZR, Sano T, Asa SL, Yamada S, Horiguchi H, Tashiro $\mathrm{T}$ ET AL Cytoplasmic expression of fibmblast growth factor receptor- 4 in human pituitary adenomas: relation to tumor type, size, proliferation, and invasiveness. J Clin Endocrinol Metab 2004; 89: 1904-11.

16. Carrasco C, Véliz J, Rojas D, Wohuk N. [Results of treatment for acromegaly in 53 patients: it is time of intervention]. Rev Méd Chile 2006; 134: 989-96.

17. Thapar K, Kovacs K, Scheithauer BW, Stefaneanu L, Horvath E, Pernicone PJ et al. Proliferative activity and invasiveness among pituitary adenomas and carcinomas: an analysis using the MIB-1 antibody. Neurosurgery 1996; 38: 99-106.

18. Trouilias J, Sassolas G, Guigard MP, Fonlupt $P$, Ansaneli-Naves L, Perrin G. Relationships between pathological diagnosis and clinical parameters in acromegaly. Metabolism 1996; 45 Suppl 1: 53-6.

19. Asa SL, Kovacs K, Horvath E, Singer W, Smyth HS. Hormone secretion in vitro by plurihormonal adenomas of the acidophil cell line. J Clin Endocrinol Metab 1992; 75: 68-75.

20. Mastronardi L, Guiducci A, Spera C, Puzzimi F, Liberati F, MaIRA G. Ki-67 labelling index and invasiveness among anterior pituitary adenomas: analysis of 103 cases using the MIB-1 monoclonal antibody. J Clin Pathol 1999; 52: 107-11.

21. TuRner HE, WASS JA. Are markers of proliferation valuable in the histological assessment of pituitary tumours? Pituitary 1999; 1: 147-51.

22. Schreiber S, SAEger W, LÜDECKe DK. Proliferation markers in different types of clinically non-secreting pituitary adenomas. Pituitary 1999; 1: 213-20.

23. Honegger J, Prettin C, Feuerhake F. Expression of Ki67 antigen in nonfunctioning pituitary adenomas: correlation with growth velocity and invasiveness. Journal of Neurosurgery 2003; 99: 674-9.

24. Dubois S, Guyetant S, Menei P, Rodien P, Illouz F, VIeLLE B ET AL. Relevance of Ki-67 and prognostic factors for recurrence/progression of gonadotropic adenomas after first surgery. Eur J Endocrinol 2007; 157: $141-7$.

25. Lndboe CF, Von Der Ohe G, Torp SH. Determination of proliferation index in neoplasms using different Ki67 equivalent antibodies. APMIS 2003; 111: 567-70. 